\title{
Ranking of Hexagonal Fuzzy Numbers for Solving Multi Objective Fuzzy Linear Programming Problem
}

\author{
Rajarajeswari. $\mathrm{P}^{1}$ \\ ${ }^{1}$ Department of Mathematics, \\ Chikkanna Government Arts College, Tirupur
}

\author{
Sahaya Sudha.$A^{2}$ \\ ${ }^{2}$ Department of Mathematics, \\ Nirmala College for Women, Coimbatore
}

\begin{abstract}
In this paper a ranking Procedure based on Hexagonal Fuzzy numbers, is applied to a Multi-objective Linear programming problem (MOLPP) with fuzzy coefficients. By this ranking method any Multiobjective Fuzzy Linear Programming problem (MOFLPP) can be converted in to a crisp value problem to get an optimal solution. This method provides an insight for the planner due to uncertain environment in an organizational Economics. In an organization, where a number of alternatives and variables such as production, inventory, financial management, costing and various other parameters are involved, this ranking procedure serves as an efficient method wherein a numerical example is also taken and the inference is given.
\end{abstract}

\section{Keywords}

Ranking, Hexagonal fuzzy numbers, MOFLPP, Decision making.

\section{INTRODUCTION}

Ranking fuzzy number is used in decision- making process in an economic environment. In an organization various activities such as planning, execution, and other process takes place continuously. This requires careful observation of various parameters which are all in uncertain in nature due the competitive business environment globally. In fuzzy environment ranking fuzzy numbers is a very important decision making procedure. The idea of fuzzy set was first proposed by Bellman and Zadeh [1], as a mean of handling uncertainty that is due to imprecision rather than randomness. The concept of fuzzy linear programming (FLP) was first introduced by Tanaka et al. [11, 12] Zimmerman [17] introduced fuzzy linear programming in fuzzy environment. Multi-objective linear Programming was introduced by Zeleny [16]. Lai Y.J -Hawng C.L [5] considered MOLPP with all parameters having a triangular possibility distribution. They used an auxiliary model and it was solved by MOLPP. Zimmerman [18] applied their approach to vector maximum problem by transforming MOFLP problem to a single objective linear programming problem. Qiu- Peng $\mathrm{Gu}$, and Bing-Yuan Cao [7] solved Fuzzy linear programming problems based on Fuzzy numbers distance. Tong Shaocheng [13] focused on the fuzzy linear programming with interval numbers. Chanas [2] proposed a fuzzy programming in multi objective linear programming. Verdegay [14] have proposed three methods for solving three models of fuzzy integer linear programming based on the representation theorem and on fuzzy number ranking method. In particular, the most convenient methods are based on the concept of comparison of fuzzy numbers by the use of ranking functions. Nasseri [6] has proposed a new method for solving FLP problems in which he has used the fuzzy ranking method for converting the fuzzy objective function into crisp objective function.

\section{PRELIMINARIES}

\subsection{Definition:}

Let $X$ be a nonempty set. A fuzzy set $A$ in $X$ is characterized by its membership function $A: X \rightarrow[0,1]$, where $\mathrm{A}(\mathrm{x})$ is interpreted as the degree of membership of element $x$ in fuzzy A for each $x \in X$.

\subsection{Interval Number:}

Let $R$ be the set of real numbers. Then closed interval $[a, b]$ is said to be an interval number, where $a, b \in R, a \leq b$.

\subsection{Distance between interval numbers:}

Let $a=\left[a_{1}, a_{2}\right], b=\left[b_{1}, b_{2}\right]$ be two interval numbers. Then the distance between $(\mathrm{a}, \mathrm{b})$ denoted by $\mathrm{d}(\mathrm{a}, \mathrm{b})$, is defined by

$$
d(a, b)=\int_{\frac{-1}{2}}^{\frac{1}{2}}\left[\left[\frac{a_{1}+a_{2}}{2}+x\left(a_{2}-a_{1}\right)\right]-\left[\frac{b_{1}+b_{2}}{2}+x\left(b_{2}-b_{1}\right)\right] d x\right.
$$

\subsection{Fuzzy number:}

A fuzzy set $\tilde{A}$ of the real line $\mathrm{R}$ with membership function $\mu_{\tilde{A}}(x): R \rightarrow[0,1]$ is called fuzzy number if

i) A must be normal and convex fuzzy set;

ii) the support of $\widetilde{A}$, must be bounded

iii) $\alpha_{A}$ must be a closed interval for every $\alpha \in[0,1]$

\subsection{Support:}

The support of a fuzzy set $\bar{A}, \mathrm{~S}(\tilde{\mathrm{A}})$, is the crisp set of all $\mathrm{x} \in \mathrm{X}$ such that $\mu_{\tilde{A}}(X) \succ 0$

\subsection{Distance between fuzzy numbers:}

Let $\tilde{A}, \tilde{B}$ be two fuzzy numbers. Then the distance between the fuzzy numbers $\tilde{A}$ and $\tilde{B}$ is defined by

$$
D(\tilde{A}, \widetilde{B})=\int_{0}^{1} d\left(\tilde{A}_{\lambda}, \tilde{B}_{\lambda}\right) d \lambda
$$

\section{HEXAGONAL FUZZY NUMBER}

A fuzzy number $\tilde{A}_{H}$ is a hexagonal fuzzy number denoted by $\tilde{A}_{H}\left(a_{1}, a_{2}, a_{3}, a_{4}, a_{5}, a_{6}\right)$ where $\left(a_{1}, a_{2}, a_{3}, a_{4}, a_{5}, a_{6}\right)$ are real numbers and its membership function $\mu \tilde{A}_{H}(x)$ is given below. 


$$
\mu_{\widetilde{\AA}_{\mathrm{H}}}(X)=\left\{\begin{array}{l}
0 \text { for } \chi<a_{1} \\
\frac{1}{2}\left(\frac{\chi-a_{1}}{a_{2}-a_{1}}\right) \text { for } a_{1} \leq \chi \leq a_{2} \\
\frac{1}{2}+\frac{1}{2}\left(\frac{\chi-a_{2}}{a_{3}-a_{2}}\right) \text { for } a_{2} \leq \chi \leq a_{3} \\
1 \text { for } a_{3} \leq \chi \leq a_{4} \\
1-\frac{1}{2}\left(\frac{\chi-a_{4}}{a_{5}-a_{4}}\right) \text { for } a_{4} \leq \chi \leq a_{5} \\
\frac{1}{2}\left(\frac{a_{6}-\chi}{a_{6}-a_{5}}\right) \text { for } a_{5} \leq \chi \leq a_{6} \\
0 \text { for } \chi>a_{6}
\end{array}\right\}
$$

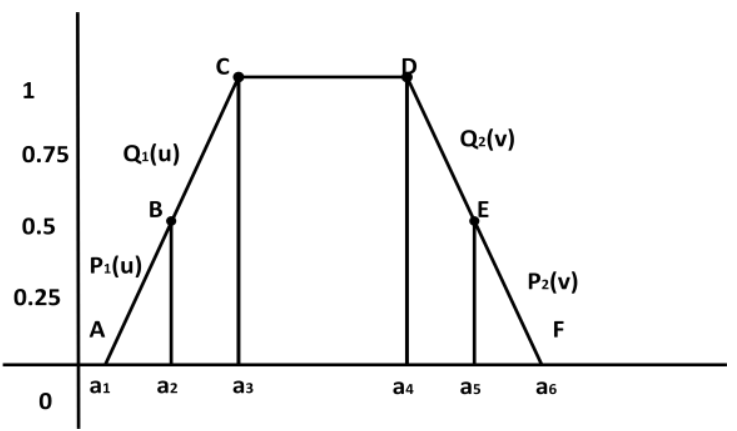

Figure 1 Graphical representation of a hexagonal fuzzy number

\subsection{Arithmetic Operations on Hexagonal Fuzzy numbers}

Let $\tilde{A}_{H}=\left(a_{1}, a_{2}, a_{3}, a_{4}, a_{5}, a_{6}\right)$ and $\tilde{B}_{H}=\left(b_{1}, b_{2}, b_{3}, b_{4}, b_{5}, b_{6}\right)$ be two hexagonal fuzzy numbers, then

(i) $\tilde{A}_{H}(+) \tilde{B}_{H}=\left(a_{1}+b_{1}, a_{2}+b_{2}, a_{3}+b_{3}, a_{4}+b_{4}, a_{5}+b_{5}, a_{6}+b_{6}\right)$

(ii) $\tilde{A}_{H}(-) \tilde{B}_{H}=\left(a_{1}-b_{1}, a_{2}-b_{2}, a_{3}-b_{3}, a_{4}-b_{4}, a_{5}-b_{5}, a_{6}-b_{6}\right)$

(iii) $\tilde{A}_{H}() \tilde{B}_{H}=\left(a_{1} * b_{1}, a_{2} * b_{2}, a_{3} * b_{3}, a_{4} * b_{4}, a_{5} * b_{5}, a_{6} * b_{6}\right)$

\subsection{Ranking of Hexagonal Fuzzy Numbers:} A number of approaches have been proposed for the ranking of fuzzy numbers. In this paper for a hexagonal fuzzy number $\widetilde{A}_{H}=\left(a_{1}, a_{2}, a_{3}, a_{4}, a_{5}, a_{6}\right)$, a ranking method is devised based on the following formula.

$$
R\left(\tilde{A}_{H}\right)=\left(\frac{2 a_{1}+3 a_{2}+4 a_{3}+4 a_{4}+3 a_{5}+2 a_{6}}{18}\right)\left(\frac{5}{18}\right)-------(1)
$$

Let $\tilde{A}_{H}=\left(a_{1}, a_{2}, a_{3}, a_{4}, a_{5}, a_{6}\right)$ and $\tilde{B}_{H}=\left(b_{1}, b_{2}, b_{3}, b_{4}, b_{5}, b_{6}\right)$

be two hexagonal fuzzy numbers then

$$
\tilde{A}_{H} \approx \tilde{B}_{H} \Leftrightarrow R\left(\tilde{A}_{H}\right)=R\left(\tilde{B}_{H}\right)
$$

$$
\begin{aligned}
& \tilde{A}_{H} \geq \widetilde{B}_{H} \Leftrightarrow R\left(\tilde{A}_{H}\right) \geq R\left(\widetilde{B}_{H}\right) \\
& \tilde{A}_{H} \leq \widetilde{B}_{H} \Leftrightarrow R\left(\tilde{A}_{H}\right) \leq R\left(\widetilde{B}_{H}\right)
\end{aligned}
$$

\section{PROPOSED RANKING ALGORITHM}

The above procedure (3.2) can be used to develop ranking for hexagonal fuzzy numbers. Based on this ranking procedure, a ranking algorithm is developed for a hexagonal fuzzy numbers. Moreover, it is applied to MOLPP under constraints with fuzzy coefficients.

\subsection{Algorithm:}

Step1: Consider the fuzzy numbers $\widetilde{A}_{H} \& \widetilde{B}_{H}$ of hexagonal fuzzy number

Step2: Find Supremum $\mathrm{M}=\operatorname{Sup}\left(\mathbf{s}\left(\tilde{A}_{H}\right) \mathrm{U} \mathrm{s}\left(\tilde{B}_{H}\right)\right)$, where

$\mathbf{s}\left(\tilde{A}_{H}\right)=$ Support set of $\tilde{A}_{H}$ and s $\left(\tilde{B}_{H}\right)=$ Support set of $\tilde{B}_{H}$

Step3: Take

$\tilde{A}_{H}=\left(a_{1}, a_{2}, a_{3}, a_{4}, a_{5}, a_{6}\right)$ and $\tilde{B}_{H}=\left(b_{1}, b_{2}, b_{3}, b_{4}, b_{5}, b_{6}\right)$ as hexagonal fuzzy numbers

Step4: Calculate

$D\left(\tilde{A}_{H}, M\right)=M-\left(\frac{2 a_{1}+3 a_{2}+4 a_{3}+4 a_{4}+3 a_{5}+2 a_{6}}{18}\right) \&$

$D\left(\tilde{B}_{H}, M\right)=M-\left(\frac{2 b_{1}+3 b_{2}+4 b_{3}+4 b_{4}+3 b_{5}+2 b_{6}}{18}\right)$

Step5: If

$$
\begin{aligned}
& D\left(\tilde{A}_{H}, M\right)>D\left(\widetilde{B}_{H}, M\right) \text { then } \tilde{A}_{H}>\widetilde{B}_{H} \\
& D\left(\tilde{A}_{H}, M\right)<D\left(\widetilde{B}_{H}, M\right) \text { then } \tilde{A}_{H}<\widetilde{B}_{H} \\
& D\left(\tilde{A}_{H}, M\right)=D\left(\tilde{B}_{H}, M\right) \text { then } \tilde{A}_{H}=\widetilde{B}_{H}
\end{aligned}
$$

Step 6: Stop.

\section{METHOD OF SOLVING MULTI- OBJECTIVE FUZZY LINEAR PROGRAMMING PROBLEM}

In this paper, we discuss a Multi-Objective Fuzzy Linear Programming Problem in constraint conditions with fuzzy coefficients. Moreover, the objectives considered in this paper are mixed with both maximization and minimization types. We discuss a model whose standard form is 
Maximize $z_{1}=c_{1} x$

Minimize $z_{2}=c_{2} x$

Subject to $\quad \tilde{A}_{H} X \leq \tilde{b}, X \geq 0$

Where $c_{i j}=\left(c_{i 1}, c_{i 2}, \ldots . c_{i n}\right)$ is an n- dimensional crisp row vector, $\widetilde{A}_{H}=\tilde{a}_{i j}$ is an m x n fuzzy matrix,

$\tilde{b}=\left(\tilde{b}_{1}, \tilde{b}_{2}, \tilde{b}_{3}, \ldots . . \tilde{b}_{m}\right)^{T}$ is an m-dimensional fuzzy line vector and $X=\left(x_{1}, x_{2}, x_{3}, x_{4} \ldots x_{n}\right)^{T} \quad$ is $\quad$ an $\mathrm{n}$-dimensional decision variable vector.

We now consider a bi-objective Fuzzy linear programming Problem with constraints having fuzzy coefficients is given by

Maximize $z_{1}=c_{11} x_{1}+c_{12} x_{2}+c_{13} x_{3}+\ldots \ldots . .+c_{1 n} x_{n}$ Minimize $z_{2}=c_{21} x_{1}+c_{22} x_{2}+c_{23} x_{3}+\ldots \ldots .+c_{2 n} x_{n}$

Subject to $\tilde{a}_{i 1} x_{1}+\tilde{a}_{i 2} x_{2}+\tilde{a}_{i 3} x_{3}+\ldots \ldots .+\tilde{a}_{i n} x_{n} \leq \tilde{b}_{i}$ $x_{1}, x_{2}, x_{3}, \ldots \ldots x_{n} \geq 0, i=1,2, \ldots \ldots . . m$ where fuzzy numbers are hexagonal, where

$\tilde{a}_{i 1}=\tilde{a}_{i 11}, \tilde{a}_{i 12}, \tilde{a}_{i 13}, \tilde{a}_{i 14}, \tilde{a}_{i 15}, \tilde{a}_{i 16}$,

$\tilde{a}_{i 2}=\tilde{a}_{i 21}, \tilde{a}_{i 22}, \tilde{a}_{i 23}, \tilde{a}_{i 24}, \tilde{a}_{i 25}, \tilde{a}_{i 26}$

$\tilde{a}_{i n}=\tilde{a}_{i n 1}, \tilde{a}_{i n 2}, \tilde{a}_{i n 3}, \tilde{a}_{i n 4}, \tilde{a}_{i n 5}, \tilde{a}_{i n 6}$,

$\tilde{b}_{i}=\tilde{b}_{i 1}, \tilde{b}_{i 2}, \tilde{b}_{i 3}, \tilde{b}_{i 4}, \tilde{b}_{i 5}, \tilde{b}_{i 6}$

By the ranking Algorithm, the above MOFLPP is transformed into a MOLPP is as follows:

Maximize $z_{1}=c_{11} x_{1}+c_{12} x_{2}+c_{13} x_{3}+\ldots \ldots .+c_{1 n} x_{n}$

Minimize $z_{2}=c_{21} x_{1}+c_{22} x_{2}+c_{23} x_{3}+\ldots \ldots . .+c_{2 n} x_{n}$

Subject to,

$2\left(a_{i 11} x_{1}+a_{i 21} x_{2}+\ldots .+a_{i n 1} x_{n}\right)+$

$3\left(a_{i 12} x_{1}+a_{i 22} x_{2}+\ldots .+a_{i n 2} x_{n}\right)+$

$4\left(a_{i 13} x_{1}+a_{i 23} x_{2}+\ldots .+a_{i n 3} x_{n}\right)+$

$4\left(a_{i 14} x_{1}+a_{i 24} x_{2}+\ldots .+a_{i n 4} x_{n}\right)+$

$3\left(a_{i 15} x_{1}+a_{i 25} x_{2}+\ldots .+a_{i n 5} x_{n}\right)+$

$2\left(a_{i 16} x_{1}+a_{i 26} x_{2}+\ldots .+a_{i n 6} x_{n}\right)$ $\leq 2 b_{i 1}+3 b_{i 2}+4 b_{i 3}+4 b_{i 4}+3 b_{i 5}+2 b_{i 6}$

$x_{1}, x_{2}, x_{3} \ldots \ldots . x_{n} \geq 0, i=1,2, \ldots \ldots . m$

Using (2), this can be converted into a single objective problem subject to the constraints with transformed crisp number coefficients and hence solved accordingly.

Similarly, multi-objective problems with more than two objectives can also be solved using the above procedure, here in the very first stage itself the problem is transformed into a crisp problem and afterwards there will be no more fuzziness in the constraints as well as in the problem.

\section{NUMERICAL EXAMPLE}

The below mentioned example is taken from Rajarajeswari. $\mathrm{P}$ and SahayaSudha A [8] in a production planning process.

$$
\begin{array}{ll}
\text { Maximize } & Z_{1}=75 x_{1}+90 x_{2} \\
\text { Minimize } & Z_{2}=60 x_{1}+75 x_{2}
\end{array}
$$

Subject to

$$
\begin{aligned}
& \widetilde{a}_{11} x_{1}+\widetilde{a}_{12} x_{2} \leq \tilde{b}_{1} \\
& \widetilde{a}_{21} x_{1}+\widetilde{a}_{22} x_{2} \leq \tilde{b}_{2} \\
& x_{1}, x_{2} \geq 0
\end{aligned}
$$

Where

$$
\begin{aligned}
& \tilde{a}_{11}=(180,190,200,200,210,220) \\
& \tilde{a}_{12}=(230,240,250,250,260,270) \\
& \tilde{a}_{21}=(280,290,300,300,310,320) \\
& \tilde{a}_{22}=(180,190,200,200,210,220)
\end{aligned}
$$

$\tilde{b}_{1}=(12000,13000,14000,14000,15000,16000)$

$\tilde{b}_{2}=(11500,12000,12500,12500,13000,13500)$

$$
\text { Maximize } Z_{1}=75 x_{1}+90 x_{2}
$$

Subject to

$(180,190,200,200,210,220) x_{1}$

$+(230,240,250,250,260,270) x_{2}$ $\leq(12000,13000,14000,14000,15000,16000)$

$(280,290,300,300,310,320) x_{1}$

$+(180,190,200,200,210,220) x_{2}$

$\leq(11500,12000,12500,12500,13000,13500)$ 


$$
x_{1}, x_{2}, x_{3}, x_{4}, x_{5}, x_{6} \geq 0
$$

By (2), this can be transformed into a crisp LPP as

$$
\text { Maximize } Z_{1}=75 x_{1}+90 x_{2} \text {, }
$$

Subject to

$2\left(180 x_{1}+230 x_{2}\right)+3\left(190 x_{1}+240 x_{2}\right)$

$+4\left(200 x_{1}+250 x_{2}\right)+4\left(200 x_{1}+250 x_{2}\right)$

$+3\left(210 x_{1}+260 x_{2}\right)+2\left(220 x_{1}+270 x_{2}\right)$

$\leq 2(12000)+3(13000)+4(14000)+4(14000)+3(15000)+2(16000)$

$2\left(280 x_{1}+180 x_{2}\right)+3\left(290 x_{1}+190 x_{2}\right)$

$+4\left(300 x_{1}+200 x_{2}\right)+4\left(300 x_{1}+200 x_{2}\right)$

$+3\left(310 x_{1}+210 x_{2}\right)+2\left(320 x_{1}+220 x_{2}\right)$

$\leq 2(11500)+3(12000)+4(12500)+4(12500)+3(13000)+2(13500)$

Case (i):

We consider the problem with the maximization objective

$$
\text { Maximize } Z_{1}=75 x_{1}+90 x_{2}
$$

Subject to

$$
\begin{aligned}
& 3600 x_{1}+4500 x_{2} \leq 252000 \\
& 5400 x_{1}+3600 x_{2} \leq 225000 \\
& x_{1}, x_{2}, x_{3}, x_{4}, x_{5}, x_{6} \geq 0
\end{aligned}
$$

The solution is $x_{1}=9.29, x_{2}=48.57$, and Max $z_{1}=5067.86$

Next we proceed to solve the problem with minimization objective

Minimize $Z_{2}=60 x_{1}+75 x_{2}$

Subject to

$$
\begin{aligned}
& 3600 x_{1}+4500 x_{2} \leq 252000 \\
& 5400 x_{1}+3600 x_{2} \leq 225000 \\
& x_{1}, x_{2}, x_{3}, x_{4}, x_{5}, x_{6} \geq 0
\end{aligned}
$$

The solution is $x_{1}=9.29, x_{2}=48.57$, and $\operatorname{Min} z_{2}=4246.43$

Case (ii): Consider the problem with the maximization objective and an additional constraint

Maximize $Z_{1}=75 x_{1}+90 x_{2}$

Subject to
$3600 x_{1}+4500 x_{2} \leq 252000$

$5400 x_{1}+3600 x_{2} \leq 225000$

$9.29 x_{1}+48.57 x_{2} \leq 5067.88$

$x_{1}, x_{2}, x_{3}, x_{4}, x_{5}, x_{6} \geq 0$

The solution is $x_{1}=9.29, x_{2}=48.57$ and $\operatorname{Max}_{z_{1}}=5067.86$

Next we proceed to solve the problem with minimization objective with additional constraint

Minimize $Z_{2}=60 x_{1}+75 x_{2}$

Subject to

$$
3600 x_{1}+4500 x_{2} \leq 252000
$$

$5400 x_{1}+3600 x_{2} \leq 225000$

$9.29 x_{1}+48.57 x_{2} \leq 5067.88$

$$
x_{1}, x_{2}, x_{3}, x_{4}, x_{5}, x_{6} \geq 0
$$

The solution is $x_{1}=9.29, x_{2}=48.57$ and $\operatorname{Min} z_{2}=4246.43$

\section{Case (iii):}

The feasibility of the solution will be explained as follows

When all the fuzzy numbers are in lesser priority values, the MOLPP becomes

Maximize $Z_{1}=75 x_{1}+90 x_{2}$,

Minimize $Z_{2}=60 x_{1}+75 x_{2}$

Subject to

$930 x_{1}+1180 x_{2} \leq 63000$
$1430 x_{1}+930 x_{2} \leq 59000$

$x_{1}, x_{2}, x_{3}, x_{4}, x_{5}, x_{6} \geq 0$

The solution is $x_{1}=13.4, x_{2}=42.82$ and

$\operatorname{Max} z_{1}=4859.64, \quad \operatorname{Min} z_{2}=4083.2$

Case (iv):

When all the fuzzy numbers are in priority values, the MOLPP becomes

Maximize $Z_{1}=75 x_{1}+90 x_{2}$, 
Minimize $Z_{2}=60 x_{1}+75 x_{2}$

Subject to

$$
\begin{aligned}
& 1600 x_{1}+2000 x_{2} \leq 112000 \\
& 2400 x_{1}+1600 x_{2} \leq 100000 \\
& x_{1}, x_{2}, x_{3}, x_{4}, x_{5}, x_{6} \geq 0
\end{aligned}
$$

The solution is $x_{1}=9.29, x_{2}=48.57$ and $\operatorname{Max} z_{1}=5067.86$, $\operatorname{Min} z_{2}=4246.43$

\section{Case (v):}

When all the fuzzy numbers are in the higher priority values, the MOLPP becomes

$$
\begin{array}{ll}
\text { Maximize } & Z_{1}=75 x_{1}+90 x_{2}, \\
\text { Minimize } & Z_{2}=60 x_{1}+75 x_{2}
\end{array}
$$

Subject to

$$
\begin{aligned}
& 1070 x_{1}+1320 x_{2} \leq 77000 \\
& 1570 x_{1}+1070 x_{2} \leq 66000
\end{aligned}
$$

\begin{tabular}{|c|c|c|c|c|c|c|c|c|}
\hline \multirow{2}{*}{\begin{tabular}{c|} 
Method \\
Objective \\
winh \\
constrints \\
\end{tabular}} & \multicolumn{4}{|c|}{ 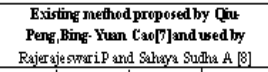 } & \multicolumn{4}{|c|}{$\begin{array}{l}\text { Proposed method } \\
\text { Hexagonal ranking }\end{array}$} \\
\hline & $x_{1}$ & $x_{2}$ & $M \propto x z_{1}$ & $\operatorname{Minz} z_{2}$ & $x_{1}$ & $x_{2}$ & $M a x z_{1}$ & $\operatorname{Minz}_{2}$ \\
\hline $\begin{array}{c}\text { Maxand } \\
\text { Min } \\
\text { objective } \\
\text { wifl same } \\
\text { constrimts }\end{array}$ & 9.29 & 4857 & 5067.86 & 4246.46 & 9.29 & 4857 & 506786 & 4246.46 \\
\hline $\begin{array}{c}\text { Maxand } \\
\text { Min } \\
\text { objective } \\
\text { with same } \\
\text { siddional } \\
\text { constraints } \\
\end{array}$ & 9.29 & 4857 & 5067.86 & 4246.46 & 9.29 & 4857 & 506786 & 4246.46 \\
\hline $\begin{array}{l}\text { Lesser } \\
\text { Priority }\end{array}$ & 7.94 & 4921 & 5023.81 & 420635 & 13.4 & 42.82 & 485964 & 4083.2 \\
\hline Prionity & 9.29 & 4857 & 5067.86 & 4246.46 & 9.29 & 4857 & 506786 & 4246.46 \\
\hline $\begin{array}{l}\text { Higher } \\
\text { Priority }\end{array}$ & 1039 & 48.05 & 5103.90 & 427922 & 5.10 & 5420 & 5260.43 & 4396.44 \\
\hline
\end{tabular}$$
x_{1}, x_{2}, x_{3}, x_{4}, x_{5}, x_{6} \geq 0
$$

The solution is $x_{1}=5.10, x_{2}=54.20$ and

$\operatorname{Max} z_{1}=5260.43, \operatorname{Min} z_{2}=4396.44$

Table 6.1: Comparison of results obtained by using existing and proposed method.

\section{RESULTS AND DISCUSSIONS}

As per the above Table (6.1), we have obtained the same results from both the existing as well as the proposed method and the feasibility of the solution is also from the lowest to higher intervals.
In the above problem the profit maximization is from [4859.64, 5260.43] and the cost of production ranges from [4083.2, 4396.44].This new method reduces the ambiguity in the solution. This forms an optimal solution for a manager to take a decision whether to produce a particular product or not, or else any alternate changes can be done .This method can be used where a particular problem cannot be solved by triangular or trapezoidal method. This method also can be used even if the number of variables and parameters are increased this method is far more efficient and easy when compared to the earlier method .This can be extended to any number of input parameters.

\section{REFERENCES}

[1] Bellman R.E and .Zadeh L.A, Decision making in a fuzzy environment, Management science 17(1970), 141164

[2] Chanas D., Fuzzy programming in multi objective linear programming-a parametric Approach, Fuzzy set and system29 (1989) 303-313.

[3] George J.klir, Boyuan, Fuzzy sets and Fuzzy logic Theory and Applications-Prentice-Hall Inc (1995) 574p

[4] Ishibuchi; Tanaka, Multi objective programming in optimization of the interval objective function, European journal of Operational Research48 (1990), 219-225

[5] Lai Y.J - Hawng C.L, Fuzzy mathematical programming, lecture notes in Economics and Mathematical systems, Springer-Verlag, (1992)

[6] S.H Nasseri,A new method for solving fuzzy linear programming by solving linear programming .Applied Mathematical Sciences, 2 (2008), 37-46

[7] Qiu-Peng Gu., Bing-Yuan Cao Approach to linear programming with fuzzy coefficients based on Fuzzy numbers distance, IEEE Transactions, 447-450. (2005)

[8] Rajerajeswari.P and Sahaya Sudha A ,Multiobjective Fuzzy Optimization Techniques in Production Planning Process ,Proc.of the Heber International conference on Applications of Mathematics and Statistics, Tiruchirappalli,India(2012),370-374

[9] Rajerajeswari.P and Sahaya Sudha A and Karthika.R A new Operations on hexagonal fuzzy number International Journal of Fuzzy Logic Systems (IJFLS) Vol.3, No3, July2013

[10] Sophiya Porchelvi R., Nagoorgani .A. Irene Hepzibah .R An Algorthimic Approach to Multiobjective Fuzzy Linear Programming Problem

[11] Tanaka H., .Asai K., Fuzzy linear programming problems with fuzzy numbers, Fuzzy Sets and Systems 13 (1984), 1-10.

[12] Tanaka H., .Okuda .T and Asai K., Fuzzy Mathematical Programming, Journal of Cybernetics and systems, 3(1973), 37-46

[13] Tong Shaocheng, Interval number and fuzzy number linear programming, Fuzzy Sets and systems 66 (1994), 301-306.

[14] Verdegay, J.L. A dual approach to solve the fuzzy linear programming problem, Fuzzy sets and Systems, 14(1984), 131-141

[15] Zadeh, L.A (1965). “Fuzzy sets.’Inf.control, 8,338-353 
[16] Zeleny, M. Multiple criteria decision making. New York: McGraw-Hill Book Company, 1982

[17] Zimmermann H.J, Fuzzy programming and linear programming with several objective functions, Fuzzy sets and system1 (1978), 45-55.
[18] Zimmermann H.J, Fuzzy mathematical programming, Computer Science \&operations Research Vol.10, No4, (1983)291-298

[19] Zimmermann H.J, (1985).Application of Fuzzy set theory to Mathematical Programming 